\title{
VARIÁVEIS HEMATOLÓGICAS DE Oreochromis niloticus COMO BIOMARCADORES DE EFEITOS PARA A EXPOSIÇÃO SUBLETAL AGUDA AO BIOPESTICIDA À BASE DE Bacillus thuringiensis.
}

Hematological variables of Oreochromis niloticus as biomarkers of effects for acute subletal exposure to bacillus thiringiensis-based biopesticide..

Variables hematológicas de Oreochromis niloticus como biomarcadores de efectos para la exposición subletal aguda a biopesticidas basado en Bacillus thuringiensis.

\section{Sandy Alves da Silva ${ }^{1}$, Wagner dos Santos Mariano, Marcelo Gustavo Paulino ${ }^{1 *}$}

${ }^{1}$ Laboratório Morfofisologia Animal Comparada, Curso de Biologia, Universidade Federal do Tocantins, Araguaína, Brasil.

*Correspondência: Coordenação do curso de Biologia-Licenciatura, Universidade Federal do Tocantins Campus Araguaína, Av. Paraguai s/n, Araguaína, Tocantins, Brasil. CEP: 77.824-838. e-mail:

marcelopaulino@uft.edu.br

Artigo recebido em 01/03/2020 aprovado em 06/11/2020 publicado em 06/11/2020

\section{RESUMO}

$\mathrm{Na}$ tentativa de controlar e combater efeitos danosos de insetos na lavoura e o impacto ambiental, os biopesticidas são alternativas biologicamente ativas que prometem causar menor dano ambiental. Os biopesticidas à base de Bacillus thuringiensis são os mais utilizados como inseticidas, entretanto, não é claro seus efeitos em organismos não-alvos. O trabalho propôs avaliar os possíveis efeitos causados por biopesticida à base de B. thuringiensis utilizando sensíveis biomarcadores hematológicos de tilápia-do-Nilo (Oreochromis niloticus). Exemplares de tilápia foram divididos em dois grupos ( $\mathrm{n}=10$ por grupo) e colocados em aquários de $150 \mathrm{~L}$. O aquário do grupo controle continha água, enquanto do grupo tratado foi adicionado $60 \mathrm{mg} \mathrm{L}^{-1}$ do biopesticida na água do aquário teste. Após exposição, $1 \mathrm{~mL}$ de sangue foi coletado, via punção caudal, para análises dos biomarcadores hematológicos: hemoglobina $(\mathrm{Hb})$, hematócrito $(\mathrm{Hct})$, eritrócitos (RBC), volume corpuscular médio (VCM), hemoglobina corpuscular média (HCM) e concentração de hemoglobina corpuscular média (CHCM), leucócitos e trombócitos totais e analise diferencial leucocitária. Após as 48 horas de exposição não houve diferença significativa nas variáveis analisadas entre o grupo controle e grupo tratado. Os resultados sugerem baixa toxicidade para a tilápia, no delineamento proposto, sem alterações que comprometessem os processos fisiológicos vitais da espécie.

Palavras-chave: Dipel, parâmetros hematológicos, ecofisiologia.

\section{ABSTRACT}

In an attempt to control and combat the harmful effects of insects on crops and the environmental impact, biopesticides are biological alternatives that promise to cause less environmental damage. Bacillus thuringiensis biopesticides are the most used as insecticides; however, their effects on non-target organisms are not clear. The work proposed to evaluate the possible effects caused by a biopesticide based on B. thuringiensis using sensitive hematological biomarkers of Nile tilapia (Oreochromis niloticus). Specimens were divided into two groups $(n=10$ per group) and placed in 150 L aquaria. The control group aquarium contained water, while the treated group added $60 \mathrm{mg} \mathrm{L^{-1 }}$ of the biopesticide to the test aquarium water. After exposure, $1 \mathrm{~mL}$ of blood was collected, via caudal puncture, for analysis of hematological biomarkers: hemoglobin (Hb), hematocrit (Hct), erythrocytes (RBC), mean corpuscular volume (MCV), mean corpuscular hemoglobin $(M C H)$ and concentration mean corpuscular hemoglobin $(\mathrm{CMCH})$, total leukocytes and thrombocytes and leukocyte differential analysis. After 48 hours of exposure, there was no significant difference in the variables analyzed between the control group and the treated group. The results 
suggest low toxicity for tilapia, in the proposed design, without alterations that would compromise the vital physiological processes of the species.

Keywords: Dipel, hematological parameters, ecophysiology.

\section{RESUMEN}

En un intento por controlar y combatir los efectos nocivos de los insectos en los cultivos y el impacto ambiental, los bioplaguicidas son alternativas biológicamente activas que prometen causar menos daño ambiental. Los biopesticidas de Bacillus thuringiensis son los más utilizados como insecticidas, sin embargo, sus efectos sobre los organismos no objetivo no están claros. El trabajo propuso evaluar los posibles efectos causados por un biopesticida basado en B. thuringiensis usando biomarcadores hematológicos de tilapia del Nilo (Oreochromis niloticus). Las tilapia se dividieron en dos grupos $(n=10)$ y se colocaron en acuarios de $150 \mathrm{~L}$. El acuario del grupo control contenía agua, mientras que el grupo tratado añadió $60 \mathrm{mg} \mathrm{L}^{-1}$ del bioplaguicida al agua del acuario. Después de la exposición, se recogió $1 \mathrm{ml}$ de sangre, mediante punción caudal, para el análisis de biomarcadores hematológicos: hemoglobina $(\mathrm{Hb})$, hematocrito $(\mathrm{Hct})$, eritrocitos $(\mathrm{RBC})$, volumen corpuscular medio (VCM), hemoglobina corpuscular media (HCM) y concentración hemoglobina corpuscular media (CHCM), leucocitos y trombocitos totales y análisis diferencial de leucocitos. Después de 48 horas de exposición, no hubo diferencias significativas en las variables analizadas entre el grupo control y el grupo tratado. Los resultados sugieren baja toxicidad para la tilapia, en el diseño propuesto, sin alteraciones que comprometan los procesos fisiológicos vitales de la especie.

Descriptores: Dipel, parámetros hematológicos, ecofisiología.

\section{INTRODUÇÃO}

A agricultura em nível mundial é uma área que traz muitos recursos e, para um bom sucesso nessa atividade, necessita-se de cuidados como aplicação de agrotóxicos para combater invasores indesejados. No âmbito da América Latina, o Brasil desponta como o maior produtor agrícola e consequentemente, maior consumidor de agrotóxicos (Kopp et al., 2007; Lopes e Albuquerque, 2018). Estes pesticidas sintéticos são classificados quanto ao organismo alvo, sua classe toxicológica, o grau de toxicidade e o grupo químico pertencente (Coutinho et al., 2005), sendo os principais os herbicidas e inseticidas de formulações químicas.

Com toda essa toxicidade dos agrotóxicos espalhados no ambiente, surgiu a produção de biopesticidas, utilizando microrganismos nas formulações comerciais, com a intenção de diminuir possíveis prejuízos a toda uma biota. A utilização de biopesticidas são comumente descritos como inertes para organismos que não são alvos específicos para o seu mecanismo de ação (Mariano, 2017).

Segundo Coutinho et al. (2005), o biopesticida mais amplamente utilizados são os que contém as subespécies da bactéria Bacillus thuringiensis $(\mathrm{Bt})$ que têm despertado um grande interesse frente aos problemas de poluição ambiental no mundo. No controle microbiano dos insetos, o entomopatógeno $\mathrm{Bt}$ oferece as melhores alternativas como bioinseticida, mostrando-se também um bom candidato à obtenção de formulações comerciais, bem como à engenharia genética de plantas (Costa et al., 2010).

Note-se que mesmo em condições de produção agrícolas muito controladas, resíduos de pesticidas podem permanecer no meio, sendo lixiviados contaminando lençóis freáticos, pondo assim em risco a saúde humana (Mariano, 2017). Como qualquer agrotóxico, o biopesticida no ambiente pode ser destinado aos corpos d'água e interagir com os organismos existentes no local.

Ecossistemas aquáticos são os mais importantes receptores finais de resíduos da agricultura e demais despejos, como industriais e domésticos. Assim, um dos objetivos principais da ecotoxicologia aquática é avaliar os riscos de agrotóxicos para a biota aquática e consequentemente para as populações humanas (Oliveira Ribeiro et al., 2006).

Revista Desafios -v. 7, Especial - PIBIC, 2020 
Organismos aquáticos são vistos como excelentes indicadores das condições ambientais para analisar a qualidade de hábitats. Particularmente, os peixes são organismos comuns e familiar nos ecossistemas aquáticos e podem refletir distúrbios em diversas escalas, devido às suas características de mobilidade, estilo de vida e por sua posição próxima do topo da cadeia alimentar (Freitas e Souza, 2009).

Refletindo efeitos do ambiente, o sangue dos peixes pertence aos grupos de biomarcadores de toxicidade, qualificando a saúde da espécie e do habitat. A hematologia, estudo das células sanguíneas, é uma importante ferramenta para auxiliar no diagnóstico e prognóstico de condições de morbidade em populações de peixes sendo que quando os animais são expostos às substâncias tóxicas, alguns parâmetros podem ser alterados (Adhikari et al., 2004).

O conhecimento da ecologia de espécies de Bacillus no solo e na água ainda não é completo (Vilain et al., 2006), sendo que, os efeitos de biopesticidas em peixes são escassos na literatura e levanta grande preocupação com o aumento da produção e uso deste agrotóxico. Diante dessa lacuna, mostra-se uma importância de estudos que forneça informações de qualidade. Diante disso, o trabalho propôs avaliar efeitos causados por biopesticida à base de $B$. thuringiensis utilizando sensíveis biomarcadores hematológicos em tilápia-do-Nilo (Oreochromis niloticus).

\section{MATERIAIS E MÉTODOS}

Animais e contaminante

Exemplares juvenis de $O$. niloticus (massa corpórea $=47,28 \pm 3,0 \mathrm{~g} ;$ comprimento total $=14,59 \pm$ $0,36 \mathrm{~cm}$ ) foram obtidos de piscicultura comercial localizada em Araguaína, TO, Brasil. Os animais foram deslocados ao Laboratório de Morfofisiologia Animal
Comparada (LaMAC - multiuso) da Universidade Federal do Tocantins, campus Cimba e aclimatados por 30 dias. Os peixes foram acondicionados em tanques de 250 L com aeração artificial constante e temperatura da água controlada em $26 \pm 2{ }^{\circ} \mathrm{C}, \mathrm{pH} 6,5$, fotoperiodo natural e alimentação diária com ração (40\% proteína), à vontade, uma vez ao dia.

O biopesticida utilizado foi em formulação comercial do $\mathrm{DiPel}^{\circledR} \mathrm{WP}$ que tem como princípio ativo esporos de B. thuringiensis, var. Kurstaki, linhagem HD-1 (mínimo de 25 bilhões de esporos viáveis por grama de produto).

\section{Delineamento experimental e coleta de amostras}

Os peixes foram divididos aleatoriamente em aquários de $150 \mathrm{~L}$ em dois grupos com 10 indivíduos por grupo, onde os parâmetros da água foram mantidos o mesmo da aclimatação. $\mathrm{O}$ aquário do grupo controle ficou livre da presença de biopesticida, enquanto o grupo tratado foi exposto a concentração de $60 \mathrm{mg} \mathrm{L}^{-1}$ do biopesticida. A exposição foi em sistema estático e de forma aguda por 48 horas. Após exposição, os animais foram anestesiados com benzocaína $\left(0,1 \mathrm{~g} \mathrm{~L}^{-1}\right)$ e em seguida ocorreu uma coleta de sangue $(0,5 \mathrm{~mL})$ obtidas via punção caudal, com o auxílio de seringas heparinizadas. O sangue foi utilizado para estudos dos parâmetros hematológicos.

O projeto foi aprovado pelo comitê de ética animal local sob o protocolo 23 101.001.738/2018-99.

\section{Biomarcadores hematológicos}

Os parâmetros hematológicos foram baseados seguindo metodologias adaptadas por (Mariano, 2017). Extensões sanguíneas foram confeccionadas e coradas com kit panótico rápido $\left(\right.$ Laborclin $^{\circledR}$ ) para análise morfofisiológica da série vermelha e branca do sangue periférico (leucogramas, eritrograma e trombograma) e 
sub-amostras de sangue foram utilizadas para determinação do hematócrito (Hct), número total de eritrócitos $(\mathrm{RBC})$, hemoglobina total $(\mathrm{Hb})$ e índices hematimétricos: volume corpuscular médio (VCM); hemoglobina corpuscular média (HCM) e concentração de hemoglobina corpuscular média (CHCM),

Para a contagem diferencial de leucócitos, foi avaliado leucócitos e trombócitos totais e a porcentagem dos diferentes tipos celulares da série branca em relação ao número total de células contadas. Para isso, foram contadas 2000 células por lâmina, analisadas em microscópio de luz (WF1 10X/18) sob magnificação de 1000 vezes.

\section{RESULTADOS E DISCUSSÃO}

Segundo a Agência de Defesa Agropecuária do Paraná (ADAPAR, 2018), as concentrações de Bt aplicados na lavoura de diversas culturas tange a média de $5 \mathrm{mg}$ Dipel por metro cúbico, sendo aplicado caldas de 400 a 700 L/ha. A concentração testada é considerada ecologicamente consistente uma vez que somente resíduos da aplicação é destinada aos corpos d'água por processos de escoamento superficial e lixiviação.

A exposição é o contato/reação entre o organismo e o composto químico, sendo que os fatores mais importantes relacionados à exposição são: o tipo, duração e frequência da exposição e a concentração do agente químico (Silva e Santos, 2007). Desta forma, a concentração usada é subletal e não causou morte ou alterações comportamentais aos indivíduos durante a exposição.

Os valores médios dos parâmetros hematológicos da série eritrocitária de tilápia do grupo controle e tratado estão representados na Figura 1. Não houve diferença significativa no $\mathrm{HCL}, \mathrm{RBC}$ e na $\mathrm{Hb}$ total entre os grupos.

Figura 1. Média \pm erro padrão da média de A. concentração de hemoglobina ([Hb]), B. hematócrito (Hct) e C. número total de eritrócitos (RBC) de O. niloticus expostas a $60 \mathrm{mg}$ $\mathrm{L}^{-1}$ de biopesticida à base de $B$. thuringiensis.
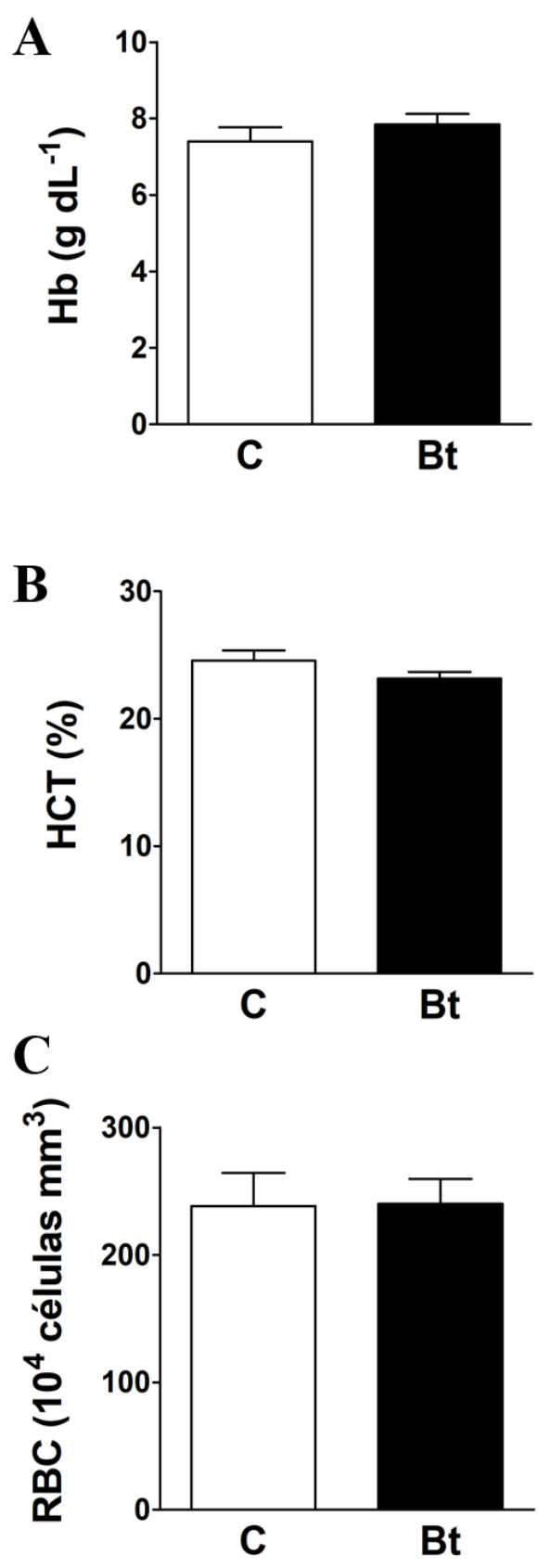

A figura 2 demonstra os valores médios dos índices hematimétricos da série vermelha do sangue. Os índices são construídos a partir dos parâmetros hematológicos e assim, também não apresentaram 
diferenças significativas entre o grupo tratado e expostos.

Figura 2. Média \pm erro padrão da média de A. concentração de hemoglobina $([\mathrm{Hb}]), \mathrm{B}$. hematócrito $(\mathrm{Hct})$ e C. número total de eritrócitos (RBC) de O. niloticus expostas a $60 \mathrm{mg}$ $\mathrm{L}^{-1}$ de biopesticida à base de $B$. thuringiensis.
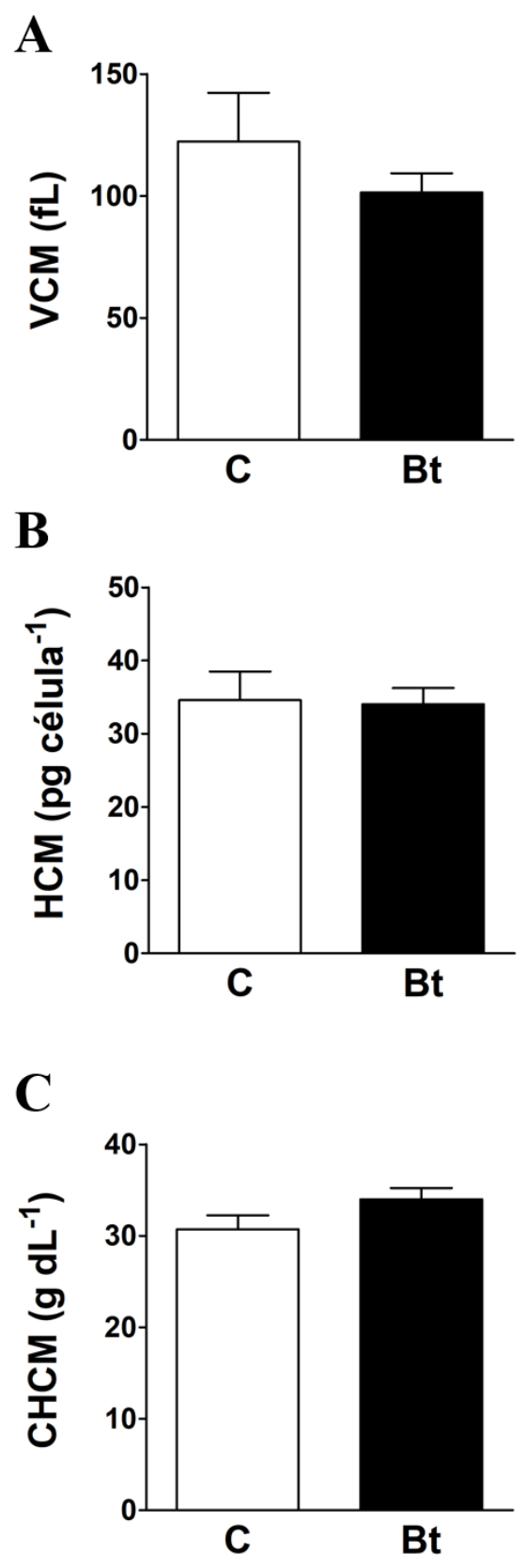

Os parâmetros sanguíneos dos peixes auxiliam na avaliação da contaminação ambiental; a análise do sangue facilita a detecção de alterações patológicas nos organismos e os desvios das condições normais do sangue observadas (Oliveira Ribeiro et al., 2000). As análises morfofisiológicas da série vermelha expressadas neste estudo, não mostrou variação relacionada ao Bt. Alterações no quadro hematológico, em nível celular, podem ser um bom indicativo de respostas fisiológicas em relação às frequentes variações ambientais (Tavares-Dias et al., 2002), assim, sugere-se que o Bt na concentração testada não é uma ameaça para a espécie.

Os resultados apresentados expressam que normalidade existente nos parâmetros hematológicos indicam que o Bt não causa quadros patológicos como anemias ou alterações celulares que dificultaria a captação e transporte de oxigênio, preservando sua integridade metabólica.

Nas extensões sanguíneos de $O$. niloticus, além da observação de eritrócitos, foram analisados os tipos celulares de leucócitos, leucócitos totais e trombócitos totais, as quais desempenham papeis importantes no organismo que sempre está exposto ao biopesticida. A figura 3 representa a extensão sanguínea dos animais do grupo controle e expostos ao biopesticida. Não houve alteração celular entre o grupo exposto e o controle (Tabela 1).

O organismo possui um sistema para combater os diferentes agentes infecciosos e tóxicos, sendo esse sistema formado pelos leucócitos e células teciduais originalmente derivados deles (Rena et al., 2001). A ativação do sistema imune poderia significar invasão do Bt ao organismo, assim, é sugerido que o pesticida não está adentrando ao sistema sanguíneo do peixe, este poderia entrar por via de ingestão, brânquias ou dérmica.

Em tilápia, neste protocolo, as vias dérmicas e via brânquias seriam sugeridas para a absorção, uma vez que os animais não se alimentaram durante a exposição. O modelo experimental $O$. niloticus é uma 
espécie de suma eficácia para uma boa resposta ao meio que esteja inserido, pois apresenta resistência a doenças virais, bacterianas e parasitárias, quando comparada a outros peixes cultivados (Popma e Lovshin, 1995).

Figura 3. Representação sanguínea de $O$. niloticus do grupo controle $(\mathrm{A}) \mathrm{e}(\mathrm{B})$ expostas a $60 \mathrm{mg} \mathrm{L}^{-1}$ de biopesticida à base de B. thuringiensis. Setas indicam linfócitos, $\mathrm{t}=$ trombócitos, e = eritrócitos. Mag. 1000x.Coloração: Kit Panótico.
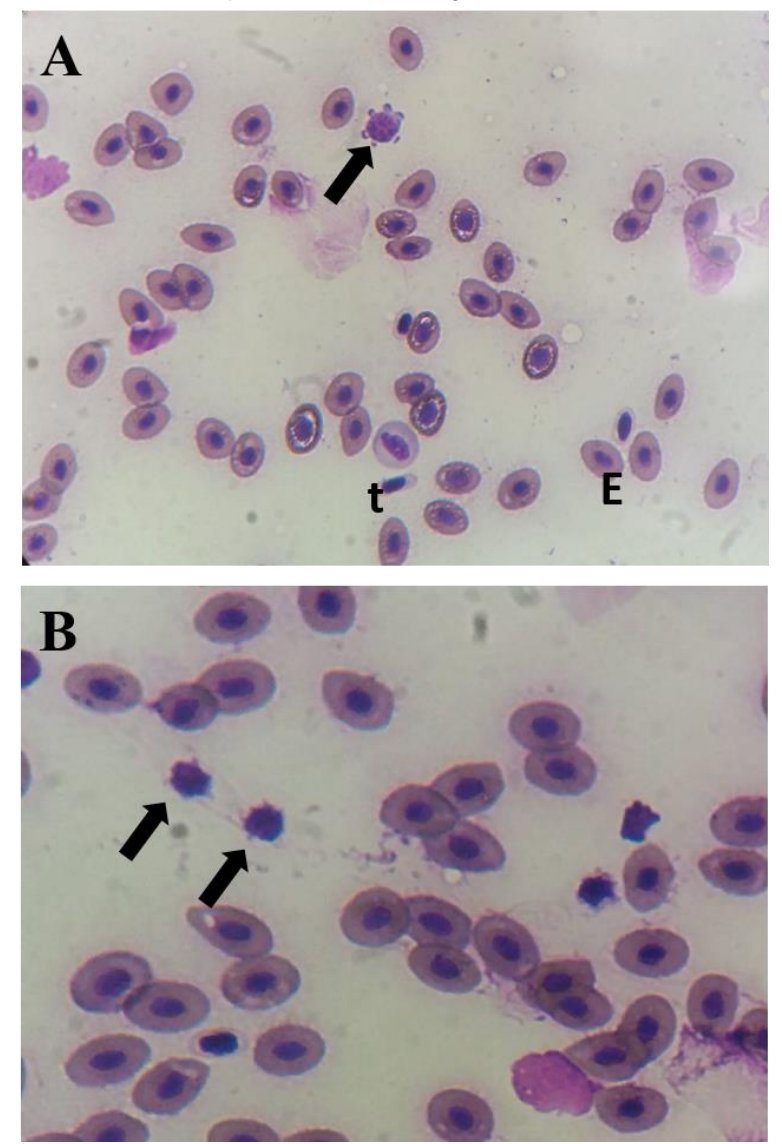

Uma das principais características fisiológicas desses animais é a sua resistência a enfermidades por patógenos, assim como a tolerância a baixos teores de oxigênio dissolvido na água (Silva et al., 2012), sendo assim, alterações no tecido sanguíneo da espécie devem ser consideradas.

No atual estudo, os peixes foram expostos a uma concentração relevante ao processo de aplicação nas lavouras. Isso pode ser um fator para que não tenha ocorrido ativação do sistema imunológico dos animais ou inativação do mesmo. O Dipel em sua formulação comercial contém $3,2 \%$ do princípio ativo Bt na sua composição. Bacillus sp. estão entre os organismos utilizados como probióticos, mesmo na aquicultura. Isso porque a capacidade de esporulação os torna resistente a diversas condições experimentais e não patogênicas e até mesmo, não toxicas quando alimentados por peixes. Assim, esse tipo de microrganismo tem ganhado atenção na manutenção da saúde e bem-estar de muitos animais aquáticos (Kuebutornye et al., 2019).

Tabela 1. Valores médios (média \pm erro padrão média) do número total de leucócitos e trombócitos e da porcentagem de linfócitos, monócitos, neutrófilos, eosinófilos, células granulocítica especial e basófilos em Oreochromis niloticus expostas ao biopesticida à base de Bacillus thuringiensis

\begin{tabular}{lcc}
\hline Tipos celulares & Controle & Bacillus thuringiensis \\
\hline Leucócitos Totais $\left(10^{3}\right)$ & $2,7 \pm 0,27$ & $3,4 \pm 0,30$ \\
Trombócitos Totais $\left(10^{3}\right)$ & $2,7 \pm 0,58$ & $2,1 \pm 0,63$ \\
Linfócitos (\%) & $59,6 \pm 7,30$ & $73,8 \pm 4,90$ \\
Monócitos (\%) & $1,0 \pm 0,18$ & $0,7 \pm 0,21$ \\
Granulocítica Especial (\%) & $0,05 \pm 0,05$ & 0,0 \\
Neutrófilos (\%) & $0,7 \pm 0,23$ & $0,3 \pm 0,11$ \\
Eosinófilos (\%) & 0 & 0 \\
Basófilos $(\%)$ & $1,0 \pm 0,27$ & $0,6 \pm 1,16$ \\
\hline
\end{tabular}


Aos bancos de dados da literatura, foi possível perceber que ainda não são conhecidas as prováveis influências e consequências que o biopesticida a base de Bt poderia causar para a fauna aquática (especificamente peixes), mesmo diante da crescente utilização de defensivos agrícolas nas culturas vegetais de produção (Mariano et al., 2015). Diante de todo estudo, possibilidades para abranger pesquisas nessa área se formam, onde ainda tem inúmeras causas para ser estudadas e descritas, surgindo um conhecimento para cuidar e proteger toda a fauna aquática.

\section{CONCLUSÃO}

O trabalho desenvolvido foi esclarecedor para o conhecimento das respostas hematológicas para a espécie Oreochromis niloticus frente a exposição subletal ao biopesticida Dipel. Ressaltando os principais biomarcadores analisados, não ocorreram alterações, mostrando baixa toxicidade do $\mathrm{Bt}$, não levando o organismo a qualquer desregulação fisiológica, indução de anemias ou deficiências metabólicas visíveis.

No sistema imunológico dos $O$. niloticus não ocorreu um aumento significativo dos glóbulos brancos, testificando que o biopesticida Bt poderia não estar sendo captado pelo organismo do animal.

\section{AGRADECIMENTO}

Os autores agradecem aos acadêmicos Alexia M. T. Simão, Ericsson R. R. Ferreira, Geraldo V.B. Dias e Ieda R.A. Ichihara na ajuda com o delineamento experimental; à técnica Luanne Pereira Gonçalves pela manutenção do laboratório.

A acadêmica Sandy Alves da Silva agradece ao apoio do Conselho Nacional de Desenvolvimento Científico e Tecnológico - CNPq - Brasil pela bolsa via Programa Institucional de Bolsas de Iniciação Científica - PIBIC, UFT.

Todos os autores declararam não haver qualquer potencial conflito de interesses referente a este artigo.

\section{REFERÊNCIAS}

ADHIKARI, S.; SARKAR, B.; CHATTERJEE, A.; MAHAPATRA, C.T; AYYAPPAN, S. Effects of cypermethrin and carbofuran on certain hematological parameters and prediction of their recovery in a freshwater teleost; Labeo rohita (Hamilton). Ecotoxicol. Environ. Saf. Vol.58, p.220 -226, 2004.

ADAPAR. Agência de Defesa Agropecuária do Paraná. 2018. Secretaria da Agricultura e do Abastecimento: DiPel ${ }^{\circledR}$ WP. Disponível em: http://www.adapar.pr.gov.br/arquivos/File/defis/DFI/ Bulas/Inseticidas/dipel.pdf. Acesso em: 07/03/2018.

COUTINHO, C.F.B.; TANIMOTO, S.T.; GALLI, A., GARBELLINI, G.S.; TAKAYAMA, M.; AMARAL, R.B.; MAZO, L.H.; AVACA, L.A.; MACHADO, S.A.S. Pesticidas: mecanismo de ação, degradação e toxidez. Pesticidas: Revista de Ecotoxicologia e Meio Ambiente, Curitiba, v. 15; 2005.

COSTA，E.L.N.; LUCHO，A.P.R.; FRITZ，L.L.; FIUZA L.M. Artrópodes e bactérias entomopatogênicos. Biotecnologia Ciência \& Desenvolvimento - $\mathrm{n}^{\circ} 38,2010$.

FREITAS, C.E.C.; SOUZA, F.K.S. O uso de peixes como indicador ambiental em áreas de várzea da bacia Amazônica. Agroambiental. v.1, n. 2; 2009.

KOPP, K; FILHO, N. R. A; ALVES, M. I. R; BASTOS, R.P. Publicações Sobre Efeitos de Pesticidas em Anfíbios no Período de 1980 a 2007. Multiciência (UNICAMP), v. 8. Campinas, p. 173186; 2007.

KUEBUTORNYE, F.K.A.; ABARIKE, E.D.; LU, Y. A review on the application of Bacillus as probiotics in aquaculture. Fish and Sellfish immunology, Apr; 87, p. 820-828; 2019.

LOPES, C.V.A.; ALBUQUERQUE, G.S.C. Agrotóxicos e seus impactos na saúde humana e ambiental: uma revisão sistemática. Saúde em 
Debate, Rio de Janeiro, v. 42, n. 117, p. 518 - 534, 2018.

MARIANO, W.S.; MORON, S.E.; GARCIA, R.G.; TAVARES-DIAS. Impactos de pesticidas e biopesticidas na aquicultura. In: TAVARES-DIAS, M.; MARIANO, W. S. (Org.). Aquicultura no Brasil: novas perspectivas. Pedro \& João Ed., São Carlos (SP). 2:621-638; 2015.

MARIANO, W.S. Efeitos de biopesticida à base de Bacillus thuringiensis na histologia e fisiologia de Piaractus mesopotamicus e Arapaima gigas. Macapá. Tese de Doutorado. Universidade Federal de Amapá - UFAP; p. 84, 2017.

OLIVEIRA RIBEIRO, C.A.; FILIPAK NETO, F.; MELA, M.; SILVA, P.H.; RANDI, M.A.F.; RABITTO, I.S.; ALVES COSTA, J.R.M.; PELLETIER, E. Hematological findings in neotropical fish Hoplias malabaricus exposed to subchronic and dietary doses of methylmercury, inorganic lead, and tributyltin chloride. Environmental Research, v. 101, p.74-80, 2006.

OLIVEIRA RIBEIRO, C.A.; PELLETIER, E.; PFEIFFER, W.C.; ROULEAU, C. Comparative uptake, bioaccumulation, and gill damages of inorganic mercury intropical and Nordic freshwater fish. Environmental Research, v. 83, p. 286-292, 2000.

POPMA, T.; LOVSHIN, L. Aspectos relevantes da biologia e do cultivo das tilápias. Panorama da Aquicultura, Rio de Janeiro, v. 5, n. 27, p. 8-13; 1995.
RENA, C.; VIDIGAL, F.M.; BARRA, A.A.; SCHELB, E.J.S.; TOLEDO, L.O.; DORNELAS, M.C.; RENA, L.R. Estudo Revisional sobre a morfologia e as funções dos Leucócitos. HU RevistaRevista Médica Oficial do Hospital Universitário da Universidade Federal de Juiz de Fora. Janeiro a Dezembro. v. 5, p. 337-344; 2001.

SILVA, R.D.; ROCHA L.O.; FORTES, B.D.A.; VIEIRA, D.; FIORAVANTE, C.C.S. Parâmetros hematológicos e bioquímicos da tilápia-do-Nilo (Oreochromis niloticus L.) sob estresse por exposição ao ar. Pesq. Vet. Bras. v. 32, p. 99-107, 2012.

SILVA, J.M.; SANTOS, J.R. Toxicologia de agrotóxicos em ambientes aquáticos. Oecol. Bras. v. 11, n. 4, p. 565-573; 2007.

TAVARES-DIAS, M; MARTINS, M.L; SCHLCH, S. H.C; ONAKA, E.M; QUINTANA, C.I.F; MORAES, R.E; MORAES, F.R. Alterações hematológicas e histopatológicas em pacu, Piaractus mesopotamicus Holmberg, 1887 (Osteichthyes, Holmberg, 1887 (Osteichthyes, Characidae) tratado com sulfato de cobre (CuSO4). Acta Scientiarum. Maringá, v. 24, n. 2, p. 547-554, 2002.

VILAIN, S.; LUO, Y.; HILDRETH, M.B.; BROZEL, V.S. Analysis of the life cycle of the soil saprophyte Bacillus cereus in liquid soil extract and in soil. Applied and Environmental Microbiology. v. 72, n. 7,: 4970- 4977; 2006. 